\title{
Interseccionalidad raza-género en las noticias digitales mexicanas sobre Kamala Harris
}

\author{
Race-gender intersectionality in Mexican digital \\ news on Kamala Harris
}

Edrei Álvarez-Monsiváis* (D) https://orcid.org/0000-0003-2779-2012

Universidad Autónoma de Nuevo León, México, edrei.alvarez@gmail.com

*Autor para

correspondencia

Recepción: 26/01/21

Aprobación: 05/04/21

Publicación: 24/05/21

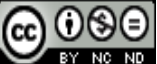

Abstract: The present study aims to analyze the news information that emerged during Kamala Harris' campaign for U.S. vicepresidency on Mexican websites. For this, a content analysis was carried out in order to detect: a) actors who intervened in the information; b) topics covered; c) racial identification of the candidate; and, d) the presence of political frames versus personal frames. $\mathrm{N}=329$ news items were selected from 10 traditional newspaper websites and 10 digital native media websites. It was found that the news focused mainly on political frames and political issues of the candidate; such behavior was more conclusive in the articles published in native digital media than in traditional media. Nonetheless, the study concludes that Harris' coverage was largely based on her race-gender intersectionality and her political ties to other men.

Key words: intersectionality, Kamala Harris, female politicians, gender studies, news frames.

Resumen: El presente estudio tiene como objetivo analizar la información noticiosa que apareció en sitios web mexicanos sobre la campaña de Kamala Harris para obtener la vicepresidencia de Estados Unidos. Para ello, se realizó un análisis de contenido de a) actores que aparecieron en la información, b) temáticas cubiertas, c) identificación racial de la candidata y d) la presencia de encuadres políticos y personales. Se seleccionaron $\mathrm{N}=329$ noticias provenientes de 10 sitios web de diarios tradicionales y 10 sitios web de medios nativos digitales. Se detectó que, en términos generales, las noticias se enfocaron en encuadres y temas políticos de la candidata, un comportamiento que resultó más contundente en las notas publicadas en medios nativos digitales que en las de los tradicionales. No obstante, el estudio concluye que la cobertura de Harris estuvo basada de manera importante en su interseccionalidad raza-género y en sus vínculos políticos con otros hombres.

Palabras clave: interseccionalidad, Kamala Harris, mujeres políticas, estudios de género, encuadres noticiosos. 


\section{Introducción}

El 11 de agosto de 2020, Joe Biden, candidato presidencial por el Partido Demócrata, anunció que Kamala Harris sería su compañera de fórmula como candidata a la vicepresidencia de Estados Unidos (Biden, 2020). Harris es politóloga y economista por la Universidad de Howard y doctora en Jurisprudencia por la Universidad de Colorado (Owens, 2016). De acuerdo con su perfil en la página web del Senado, su carrera política incluye haberse desempeñado como fiscal del distrito de San Francisco de 2003 a 2011 , además de ser electa como fiscal general de California en las contiendas de 2010 y 2014. En 2017 prestó juramento como senadora de aquel estado, lo cual la convirtió en la segunda mujer afroamericana y primera de Asia del Sur en la historia del Senado. Harris también había realizado campaña para la presidencia del país durante las elecciones primarias demócratas, pero la interrumpió el 4 de diciembre de 2019 por falta de recursos económicos (Mars, 2019).

El 14 de diciembre de 2020, el Colegio Electoral confirmó a Biden y a Harris como ganadores de la elección presidencial (Cordero, 2020); así, Kamala se convirtió en la primera mujer vicepresidenta de Estados Unidos y la primera persona de raza no blanca en conseguir el puesto, ya que es descendiente del jamaiquino Donald Harris y de la indio-americana Shyamala Gopalan (Barry, 2020). En el plano personal, Kamala está casada con el abogado estadounidense Douglas Emhoff y es madrastra de dos hijos, Ella y Cole (Lyall, 2020).

En este contexto, la presente investigación tiene como objetivo analizar la información noticiosa que apareció durante la campaña a la vicepresidencia de Kamala Harris en sitios web mexicanos. Este trabajo se inserta en los estudios sobre interseccionalidad, la cual es definida como un enfoque crítico que permite comprender las desigualdades que enfrentan aquellas identidades producto de las conexiones entre género, raza, etnia y clase social (García-Peter y Villavicencio-Miranda, 2016; Guzmán Ordaz, 2015). Dicho enfoque surgió durante la tercera ola del feminismo en la década de 1990, particularmente del feminismo negro, con el fin de explorar y entender las experiencias racistas y sexistas que viven las mujeres afrodescendientes (Alexander-Floyd, 2014; Collins, 1990; Crenshaw, 1991). Para Guzmán Ordaz (2015), la propuesta de la interseccionalidad consiste en cuestionar la visión esencialista y estática de la categoría mujer empleada por los feminismos hegemónicos, para dar pie a una teoría que no presente al género, raza y clase social como elementos disociados ni disyuntivos, sino entendidos de forma simultánea. 
$\mathrm{Al}$ estar caracterizada la identidad de Kamala Harris como mujer y no blanca, el presente trabajo busca reconocer el abordaje mediático a partir de una mirada interseccional entre raza y género, lo cual es recomendable para identificar narrativas comunicativas complejas y a menudo entrelazadas de manera opaca que no se obtendrían con el estudio de una sola categoría (Brown y Gershon, 2016; Galy-Badenas y Gray, 2020; Towner y Clawson, 2016). Para ello, se propone una exploración al estado del arte de la cobertura periodística sobre mujeres políticas y, posteriormente, a los antecedentes relacionados con el abordaje de mujeres con identidades interseccionales.

\section{Estado del arte}

\section{Cobertura periodistica de mujeres politicas}

Los hallazgos que suelen encontrarse en los estudios que abordan una diferencia entre el abordaje de candidatos y candidatas políticas se pueden condensar en cuatro líneas de investigación: mediatización de su esfera íntima, visibilidad informativa, atributos de personalidad y agenda temática. Para el primer caso, se ha explorado que los medios suelen reparar en la vida privada de las políticas al mencionar a sus hijos, padres, esposo, anécdotas personales y rutinas domésticas o de cuidado (Flores Márquez, 2020; Foster Shoaf y Parsons, 2016; García Beaudoux et al., 2018; Marañón Lazcano et al., 2018; Rista, 2020; Van der Pas y Aaldering, 2020). Para Rodelo (2020), con esta actitud los medios atribuyen a las candidatas una habilidad para las labores domésticas, lo cual les dificulta ser tomadas en cuenta en la arena política.

En este mismo tenor, se observa una insistencia de los medios por enfocarse en la apariencia y vestimenta de las candidatas políticas. Como ejemplos recientes están los estudios sobre: Hillary Clinton en la campaña presidencial 2016 en Estados Unidos, María Eugenia Vidal para la alcaldía de Buenos Aires (García Beaudoux et al., 2018), Josefina Vázquez Mota para las presidenciales de 2012 en México (Vidal Correa, 2020a), Wendy Davis para la gubernatura de Texas en 2014 (Waters et al., 2018) y Cristina Fernández para la vicepresidencia de Argentina en 2019 (Rista, 2020). Algunos atributos como ser llamativas, glamurosas, exóticas, a la moda, con cuerpos producto de cirugías y comparaciones con muñecas Barbie han sido registrados en los antecedentes de investigación (Foster Shoaf y Parsons, 2016; Rincón Soto, 2015; Waters et al., 2018). Este tipo de prácticas mediáticas objetiva a las mujeres (Rodelo, 2020), además de reproducir y naturalizar estereotipos que podrían funcionar como atajos cognitivos para que el electorado decida su voto (García Beaudoux et al., 2020). 
En cuanto a visibilidad mediática de las campañas femeninas, se han detectado tres hallazgos. El primero es que la cantidad de información que surge sobre las campañas de mujeres suele ser menor que la de los hombres, de acuerdo con investigaciones en diversos países (Haraldsoon y Wängnerud, 2019; Flores Márquez, 2020; Rodelo, 2016 y 2020; Van der Pas y Aaldering, 2020; Vidal Correa, 2020b). También se observa que las mujeres aparecen menos en los encabezados de las noticias (Vidal Correa, 2020a, 2020b), y que son invitadas en menor medida a entrevistas periodísticas que los hombres (Baitinger, 2015; García Beaudoux et al., 2020; Mitchelstein et al., 2019).

El segundo hallazgo en términos de visibilidad es que las candidatas no suelen tener el protagonismo completo en las piezas informativas (Flores Márquez, 2020), sino que se les aborda junto con hombres influyentes para brindar legitimidad a su campaña (García Beaudoux et al., 2018). Ríos Sierra (2017) denomina a esta actitud de los medios como corresponsabilidad, la cual, de forma reiterada, demerita los logros de las candidatas y los hace parecer como que fueron alcanzados por su relación con alguien más, en detrimento de dar a conocer los propios títulos y trayectorias de las mujeres (Power, 2017; Rista, 2020).

Una última línea de investigación sobre visibilidad ha reparado en lo novedoso que resulta para los medios que una mujer contienda para un puesto político (Lima y Panke, 2016). Se ha registrado que las noticias hacen referencias explícitas acerca del sexo de las candidatas, esto es, hay una insistencia por presentar palabras como mujer, mujeres, candidata femenina, entre otras (Van der Pas y Aaldering, 2020; Vidal Correa, 2020a; Ward, 2016a). Para Lachover (2017), esta acción de reiterar el género no tiene mayor valor noticioso que el de remarcar la otredad de las candidatas. Además, el hecho de que una mujer participe en la arena política resulte poco común, ampara la idea de que las mujeres no pertenecen a dicho ámbito (Rodelo, 2020).

Los estudios también han señalado que los medios encuadran la personalidad de las mujeres políticas. En ese sentido, se ha encontrado una dicotomía en la información noticiosa para mostrarlas, desde una personalidad femenina, por medio de rasgos como la sensibilidad, simpatía y compasión (García Beaudoux et al., 2018; Rodelo, 2020; Waters et al., 2018), o por medio de rasgos masculinos como severidad, ambición, frialdad y distancia (Panke, 2015; Quevedo Redondo y Suárez-Romero, 2017; Waters et al., 2018). Hay una tendencia hacia la masculinización de los atributos de las candidatas, tanto en sus spots (Panke et al., 2015), como en la cobertura mediática (Álvarez-Monsiváis, 2020; Van der Pas y Aaldering, 2020). Para Gerrits et al. (2017), la masculinización excluye a las mujeres y a la feminidad del 
ámbito político, y reafirma la hegemonía de los hombres y la masculinidad en dicho espacio.

En relación con la agenda temática, los tópicos de cuidado asociados con lo femenino, como educación, bienestar social, salud, transporte y cultura, fueron los más localizados en las notas sobre candidatas políticas (Van der Pas y Aaldering, 2020; Waters et al., 2018), en detrimento de temáticas relativas a lo masculino, como economía, seguridad y política exterior (Humprecht y Esser, 2017), a pesar de que la agenda de las candidatas esté abocada de manera preferente a temas masculinos (Kwon y Frisby, 2015). Dicha conducta de los medios de comunicación se presenta como un desbalance en cuanto a género, ya que limita el campo de acción de las candidatas (Álvarez-Monsiváis, 2019 y 2020).

\section{Cobertura periodistica de mujeres con identidad interseccional}

De acuerdo con Brown y Gershon (2020), la investigación académica sobre mujeres políticas se ha centrado de forma histórica en las de raza blanca, lo cual ha restringido el conocimiento colectivo. Sin embargo, algunas investigadoras han analizado la cobertura informativa de mujeres con identidades interseccionales.

Respecto a la mediatización de la esfera íntima, se suele encontrar el mismo fenómeno de informar acerca de la vida personal de las candidatas de minorías. Por ejemplo, la cobertura de la campaña de la representante republicana afrodescendiente Mia Love fue realizada desde estereotipos de género, como la madre amorosa y la esposa devota a su marido blanco (Wineinger, 2019). En lo relacionado con la vestimenta y apariencia, Bashri (2019) detectó que el atuendo por el cual demuestran su fe las candidatas musulmanas acapara la atención de la prensa, principalmente, al inicio de las campañas.

En la cuestión de visibilidad, se ha encontrado que el número de piezas periodísticas acerca de candidatas interseccionales es aún menor que el de mujeres blancas (Evans, 2015; Gershon, 2012; Ward, 2016a y 2016b). Únicamente las mujeres con identidad interseccional postuladas por partidos conservadores tienen un mayor espacio en los mensajes informativos por la novedad que suponen (Snipes y Mudde, 2019; Ward, 2016b).

En lo concerniente al género de las candidatas en las notas, se tiene evidencia de que la información vinculada con las candidatas de identidad interseccional es explícita no sólo en género, sino también en raza (GalyBadenas y Gray, 2020; Lucas, 2017; Towner y Clawson, 2016; Ward, 2016a 
y 2016b); mientras que la raza y el sexo de los candidatos blancos resultan incuestionables para los medios (Ward, 2016a). Además, la reiteración del género es hasta cinco veces más evidente en la información noticiosa sobre mujeres de origen étnico que en el caso de las mujeres blancas, a través de frases como "primera mujer musulmana", "primera mujer asiática", "primera mujer afrodescendiente” (Ward, 2016a y 2016b). En ese sentido, la intersección entre género, raza y religión captura la atención de los medios; sin embargo, ello le resta legitimidad a sus puestos, pues pareciera que las candidatas ingresan por cuota racial y no por su experiencia política (Galy-Badenas y Gray, 2020). Además, con estas frases, los medios refuerzan la idea de centrar los criterios de votación en las identidades de las candidatas (Lucas, 2017).

Respecto a la personalidad de las mujeres políticas, se ha observado que las políticas afrodescendientes han tenido que redefinir la manera en que son percibidas por los medios y la ciudadanía, para romper con ciertos estereotipos y proponer una nueva forma de teoría del liderazgo (Johnson Carew, 2016; Showunmi et al., 2016). Por ejemplo, Michelle Obama era vista como una mujer fría, seria y voluble al inicio de la campaña presidencial de Barack Obama, por lo cual tuvo que reconstruir su imagen, mostrando en videos de YouTube su lado materno y promoviendo el sueño americano a través de un lenguaje más afectuoso (Meyers y Goman, 2017). Con el paso del tiempo, Michelle se convirtió en un modelo a seguir, pero también en un estereotipo de lo que los votantes blancos esperan de las políticas afrodescendientes (Block Jr., 2017; Haynes y Block Jr., 2019). Otro ejemplo es la política de origen asiático que aspiraba a ser la alcaldesa de Toronto, Olivia Chow, quien debió lidiar con una cobertura periodística que la reflejó como poco empática (Maiolino, 2018).

En cuanto a la agenda temática de las noticias sobre mujeres interseccionales, se ha encontrado que existe una percepción mediática de que las mujeres afrodescendientes suelen ser más liberales que las de otras razas y géneros (Johnson Carew, 2016), y que ellas tienen una agenda en apoyo a las comunidades a las cuales pertenecen (Gershon y Lavariega Monforti, 2019). Por tal motivo, los medios esperan que su campaña esté volcada hacia cuestiones raciales y trabajen en políticas compensatorias para la inserción de minorías raciales en los grupos de poder (Maiolino, 2018). Debido a esta falsa percepción liberal, las afroamericanas postuladas por partidos conservadores suelen endurecer su posición ante temas como inmigración o del nacionalismo excepcional para ganar terreno entre sus votantes (Wineinger, 2019). Una agenda acorde a la raza y etnia acorta el nivel de acción de las 
candidatas; al mismo tiempo que desentiende a los representantes blancos de hacerse cargo de este tipo de temáticas (Block Jr., 2019; Lucas, 2017).

El impacto de dichos estereotipos mediáticos ha sido estudiado de diversas maneras, por ejemplo, al observar el tono de las notas y la intención del voto. Sobre el tono de la información, se ha evidenciado que las mujeres blancas han obtenido una cobertura menos negativa con el paso del tiempo (Van der Pas y Aaldering, 2020; Vidal Correa, 2020b; Wagner et al., 2019). Sin embargo, esto no parece aplicar para las notas informativas sobre mujeres de origen diferente al blanco, cuyo tono negativo permanece constante (Gershon, 2012; Ward, 2016a y 2016b). Para explicar este comportamiento, Tolley (2015) registró que los medios suelen hacer mayor énfasis en su inexperiencia y ser más juiciosos en los errores cometidos, a pesar de que sus contrapartes hombres o mujeres blancas tengan una trayectoria política similar (Towner y Clawson, 2016).

Los estudios sobre intención del voto para candidatas de origen étnico y racial han demostrado que generalmente éstas obtienen un mayor número de opiniones favorables entre la comunidad identitaria a la cual pertenecen (Mogahed y Mahmood, 2019). En contraparte, se ha revelado que hay un colorismo en la política electoral, ya que votantes blancos prefieren elegir a candidatos con tonos de piel más claros, mientras que son mayormente críticos al momento de decidir votar por una persona de raza negra (Block Jr., 2019).

Con base en lo anterior, se establecieron cuatro preguntas de investigación:

PI1. ¿Cuáles actores aparecieron en las noticias web mexicanas que cubrieron la campaña electoral de Kamala Harris?

PI2. ¿Qué temáticas abordaron las noticias recolectadas que hacen referencia a Harris?

PI3. ¿De qué manera se retrató el origen racial de Kamala en la información digital recolectada?

PI4. ¿Qué diferencias hubo entre los encuadres de género, raza, novedad, experiencia política y candidata?

Para esta última pregunta de investigación se conformaron dos hipótesis, con el fin de medir las diferencias entre dichos encuadres:

H1. Los encuadres personales relacionados con el género, la raza y la novedad dominaron la cobertura de la campaña de Kamala Harris en la prensa digital, en detrimento de los encuadres políticos (experiencia política y candidata). 
H2. Los diarios tradicionales que publicaron noticias en sus sitios web recurrieron en mayor medida a los encuadres políticos que a los medios nativos digitales.

\section{Estrategia metodológica}

Para responder a las preguntas de investigación, se realizó un análisis de contenido en su aproximación inductiva por medio de un programa computacional, como el efectuado por Aaldering y Vliegenthart (2016). Para Arbeláez Gómez y Onrubia Goñi (2014), una aproximación inductiva del análisis de contenido otorga mayor libertad para que sea el propio texto el que indique cuáles son las palabras clave, frases, tópicos y atributos más reiterados en todo el texto, con el fin de formular inferencias pero sin perder los valores de sistematicidad, verificación y reproducción de resultados que distinguen a la técnica (Bernete, 2013; García Galera y Berganza Conde, 2005). En ese sentido, los códigos son extraídos del mismo texto sin clasificaciones previas, por lo que es necesario fragmentarlo y agruparlo en categorías que estarán en constante redefinición conforme se analicen uno a uno los documentos (González-Teruel, 2015).

El análisis de contenido resultó relevante para esta investigación porque fue la misma técnica que se usó en estudios precedentes sobre candidatas políticas en la prensa mexicana (Flores Márquez, 2020; Rodelo, 2016 y 2020; Vidal Correa, 2020a, 2020b y 2020c), latinoamericana (García Beaudoux et al., 2020; Mitchelstein et al., 2019), norteamericana (Wagner et al., 2019; Waters et al., 2018) y europea (Humprecht y Esser, 2017; Lachover, 2017; Sensales et al., 2016), así como en comparativas internacionales de cobertura (Álvarez-Monsiváis, 2020; García Beaudoux et al., 2018; Haraldsson y Wängnerud, 2019). De igual forma, es una técnica probada en los análisis sobre políticas con identidades interseccionales (Bashri, 2019; Snipes y Mudde, 2019; Tolley, 2015; Ward, 2016a y 2016b). Además, la elección del análisis de contenido se sustenta por ser el método más empleado en los estudios de comunicación política (Piñeiro-Naval y Morais, 2020).

Asimismo, se usó el modelo del encuadre noticioso, el cual se centra en las diferentes "modalidades de mensajes escritos, hablados, gráficos y visuales que los periodistas utilizan para contextualizar un evento, tema y/o actores dentro de una o más noticias" (D’Angelo y Shaw, 2018: 214). La base del modelo consiste en que los periodistas seleccionan aspectos de una realidad percibida para reproducir un mensaje con conceptos e interpretaciones específicas (Ardèvol-Abreu, 2015; Piñeiro-Naval y Mangana, 2018). Los 
encuadres han sido utilizados para analizar noticias sobre candidatas políticas (Álvarez-Monsiváis, 2019 y 2020; Quevedo Redondo y Suárez Romero, 2017; Rista, 2020; Vidal Correa, 2020a, 2020b y 2020c; Waters et al., 2018) y con identidades interseccionales (Snipes y Mudde, 2019; Ward, 2016a, 2016b), lo que vuelve al framing pertinente para el estudio. También se han encontrado trabajos de meta-investigación en los que se evidencia la relevancia del estudio de los encuadres a partir del análisis de contenido (Matthes, 2009; Carrasco-Campos y Saperas, 2015).

Para la selección de los medios, se decidió que debían ser mexicanos y que publicaran noticias políticas en una página web de gran alcance. De esta manera, el enfoque del actual estudio es revelar una mirada transnacional de cobertura que explique cómo los medios construyen realidades e identidades sobre eventos internacionales (García Galindo y De Vicente Domínguez, 2014; Quevedo Redondo y Suárez Romero, 2017; Rodríguez-Wangüemert et al., 2019).

Para comprobar lo encontrado por Nielsen (2020), quien detectó que los medios tradicionales tienen un mejor tratamiento respecto a las candidaturas interseccionales, al cubrir sus demandas y ampliar sus problemáticas, se decidió analizar tanto medios digitales que surgieron a partir de una versión impresa (tradicionales) como medios nativos digitales. De los primeros, se seleccionaron los diez que registraron un mayor promedio mensual de visitantes, de acuerdo con el Catálogo Nacional de Medios Impresos del Instituto Nacional Electoral (INE, 2020), los cuales fueron: Milenio (2021), El Heraldo (2021), El Universal (2021), Excélsior (2021), La Jornada (2021), El Financiero (2021), El Sol de México (2021), Reforma (2021), 24 Horas (2021) y Publimetro (2021) (Tabla 1$){ }^{1}$

Respecto a los diez medios nativos digitales considerados para estudio, se eligieron: Animal Politico (2021), Aristegui Noticias (2021), SDP Noticias (2021), Sin Embargo (2021), UnoTV (2021), La Silla Rota (2021), Reporte Índigo (2021), Sopitas (2021), López Dóriga (2021) y Cultura Colectiva (2021). De estos diez sitios web, nueve son los medios de información política más visitados, según el informe de Comscore para El Economista (Merchant, 2020). Cabe señalar que se agregó Reporte Índigo para equiparar la cifra con la de los medios tradicionales, y también porque reportó 3.3 millones de visitantes de acuerdo con el INE (2020).

Para conformar el corpus de noticias, se realizó un censo de éstas del $10 \mathrm{de}$ agosto al 8 de noviembre de 2020; este periodo abarca desde el día anterior a que Joe Biden anunciara a Kamala Harris como su candidata a la vicepresidencia

1 Todas las tablas se encuentran en el Anexo, al final del presente artículo (Nota del editor). 
hasta un día posterior a que las agencias AFP y Reuters (2020), entre otros medios, comunicaran que los ganadores de la elección eran los candidatos del Partido Demócrata. Ya que la presente investigación se centra en la forma de abordaje hacia Kamala Harris, su nombre debía aparecer en el título de la noticia, para asegurar que ella era la protagonista de la información (ÁlvarezMonsiváis, 2020; Catalán-Matamoros y Peñafiel-Saiz, 2019; Bleich et al., 2015). La recopilación de los mensajes se realizó con la búsqueda avanzada de Google Noticias, con base en los criterios anteriormente expuestos, para conformar una muestra de $N=329$ noticias, distribuidas en $n=222$ para las noticias web de los medios tradicionales y $n=107$ para las de medios digitales (Tabla 1).

Una vez recolectadas las notas periodísticas, los pasos a seguir para analizarlas se derivaron de una adaptación a la propuesta de análisis de contenido cualitativo de Díaz Herrera (2018). El primer paso fue recuperar las noticias a través de la extensión de Google Chrome, llamada NCapture, cuya función fue guardar de manera automática la noticia y excluir cualquier dato externo a la información, como comentarios, publicidad, notas relacionadas, barras de navegación, entre otros elementos que no formaban parte estrictamente del mensaje. El segundo paso fue configurar las categorías; para ello se utilizó el software NVivo, el cual analiza información no estructurada con el fin de generar categorías para organizar datos cualitativos. Este programa detectó las 329 noticias guardadas por Ncapture, y a través del resumen y nube de palabras registró las mil palabras (con una extensión de al menos tres letras) más repetidas en el conjunto de textos.

El tercer paso fue crear el árbol de categorías con base en las palabras más reiteradas, en el cual se agruparon en categorías mayores con la intención de hacer un análisis más fino para explorar el contenido latente del texto. Si bien se trató de una perspectiva inductiva, se crearon grandes categorías según las preguntas de investigación: actores, temáticas y formas de mencionar el origen racial. Para el caso de la última pregunta, se conformaron cinco encuadres de forma inductiva como lo recomendó Muñiz (2020):

- Encuadre de género: cantidad de noticias en las que apareció la palabra "mujer" o afines, en referencia a Kamala Harris.

- Encuadre de raza: cantidad de notas en las que se mencionó el origen racial de Harris.

- Encuadre de novedad: número de documentos en los que apareció la palabra "primera" para señalar que se trataba de la primera mujer y/o afrodescendiente en optar o ganar el puesto. 
- Encuadre de experiencia política: número de textos en los cuales se presentó al menos un antecedente político de Harris, como: su cargo en el Senado, su puesto como fiscal general del estado de California y como fiscal de distrito.

- Encuadre de candidata: cantidad de noticias en las que se mencionó a Harris por ser candidata, aspirante o nominada a la vicepresidencia de Estados Unidos.

El cuarto paso consistió en validar las categorías y hacer una recategorización; por lo tanto, del conteo de palabras, se depuraron aquellas que no describían la categoría creada. Por ejemplo, una noticia era válida si contenía la palabra "negra" para aludir a la raza de Kamala Harris, pero era eliminada si se refería a otra persona o al color de un objeto. Asimismo, se refinaron las categorías existentes y se crearon nuevas de acuerdo con las palabras que tenían relevancia. Como último paso, y producto de los procedimientos anteriores, mediante NVivo se generó un informe donde se registró el conjunto de categorías con su nivel de frecuencia, por medio del cual se hicieron los análisis estadísticos pertinentes. Al ser un software el que arrojó los datos, no se realizó una prueba de fiabilidad intercodificadora.

Como se puede observar, la estrategia metodológica obedeció a una técnica automatizada de análisis de contenido. De acuerdo con el modelo de Boumans y Trilling (2016), la aproximación utilizada consistió en un método de conteo basado en diccionarios (Counting and Dictionary), al buscar el número de ocasiones que aparecieron distintas palabras clave con ayuda de un programa computacional. En ese sentido, no se programó un algoritmo que efectuara el análisis como en la aproximación supervisada (Supervised Machine Learning), ni tampoco se realizaron análisis semánticos de co-ocurrencias como en la no supervisada (Unsupervised Machine Learning). Sin embargo, nuestra estrategia metodológica comparte con esta última aproximación que la revisión de los textos fue de manera inductiva y abierta.

\section{Análisis de resultados}

Para responder a la primera pregunta de investigación referente a quiénes aparecieron en la información periodística digital junto con Kamala Harris, se realizó una lista de actores con sus medias, las cuales indican la proporción del total de noticias en las que cada personaje estuvo presente (Tabla 2). De acuerdo con los resultados, hubo una constante en mostrar a la candidata acompañada de al menos un personaje más $(M=0,99)$, quienes fueron, principalmente, hombres. Los más mencionados resultaron ser: su compañero 
de fórmula Joe Biden $(M=0.95)$, el actual presidente de Estados Unidos, Donald Trump $(M=0,80)$ y, en menor medida, su homólogo del Partido Republicano, Mike Pence $(M=0,33)$. Algunas mujeres que se destacaron en la cobertura fueron: la ex candidata presidencial Hillary Clinton $(M=0,14)$, la ex candidata a la vicepresidencia, Sarah Palin $(M=0,07)$ y la periodista y moderadora del debate presidencial, Susan Page $(M=0,07)$. Aunque con una participación discreta, también se mencionó a algunos familiares de Harris: su esposo Douglas Emhoff $(M=0,08)$, su hermana Maya Harris $(M=0,06)$ y su madre Shyamala Gopalan $(M=0,06)$.

Respecto a la segunda pregunta de investigación relacionada con los temas de las noticias (Tabla 3), el principal tópico estuvo asociado con la coyuntura de la pandemia de Covid-19 $(M=0,52)$. Los temas de política internacional y migración $(M=0,44)$, racismo $(M=0,42)$ y economía $(M=0,33)$, que suelen ser considerados como masculinizados, se registraron en una mayor cantidad de notas en detrimento de tópicos asociados con las labores de cuidado o feminizados, como derechos civiles y minorías $(M=0,30)$, ciencia $(M=0,08)$ y cambio climático $(M=0,08)$. Asimismo, se encontraron noticias sobre los eventos políticos típicos de la campaña como son debates electorales $(M=0,40)$, resultados de la elección $(M=0,33)$ y la cobertura en torno a la convención demócrata $(M=0,13)$.

En cuanto a la tercera pregunta de investigación, se detectó que no hubo una sola forma de mencionar los orígenes raciales de Kamala Harris (Tabla 4). La principal fue al describir el origen de su padres $(M=0,84)$, debido a su ascendencia jamaiquina por parte de su padre e india por su madre. También se le referenció desde términos coloristas, ya que fue mencionada como negra en la mitad de las noticias $(M=0,50)$ y con las diferentes combinaciones de la palabra "afro" $(M=0,44)$, como afroestadounidense o afrodescendiente. Cabe señalar que sus orígenes asiáticos $(M=0,31)$ y específicamente indios $(M=0,17)$ estuvieron más presentes que su raza jamaiquina $(M=0,04)$.

Para responder a la última pregunta de investigación, se realizó un seguimiento de los encuadres de: candidata, novedad, género, raza y experiencia política, cuyos resultados se encuentran en la Tabla 5. Para saber si existían diferencias significativas entre los encuadres, se efectuó un análisis de varianza (ANOVA) de los resultados obtenidos; así se detectaron diferencias estadísticamente significativas entre los frames, $F(1069)=28.708, p<0,001$.

Después se realizó una prueba post hoc basada en la distribución $t$ de Student y la corrección de Bonferroni. Ello permitió determinar la existencia de tres grupos entre los frames. Así, el encuadre de candidata $(M=0,87$, $D E=0,33)$ fue el que mayormente se presentó en los medios analizados, 
seguido del encuadre de experiencia política $(M=0,68, D E=0,47)$. En un tercer lugar se situaron los encuadres de género $(M=0,60, D E=0,49)$, raza $(M=0,57, D E=0,50)$ y novedad $(M=0,53, D E=0,50)$, entre los cuales no se detectaron diferencias estadísticas. Con base en estos resultados, fue posible rechazar la $H 1$ que aseveraba que los encuadres de género, raza y novedad serían más frecuentes que los de experiencia política y candidata.

Asimismo, se buscó analizar si existían diferencias entre los medios nativos y tradicionales. A través de la prueba $\mathrm{d}$ de Cohen, se encontró que el tamaño del efecto fue mayor, aunque no contundente, entre el encuadre político presentado por los medios nativos $(M=0,75, D E=0,44)$ y el de los medios tradicionales $(M=0,65, D E=0,48)$; de igual forma, el efecto fue mayor entre el encuadre de candidata de los nativos $(M=0,92$, $D E=0,28)$ y el de los tradicionales $(M=0,85, D E=0,36)$. Por lo tanto, se comprobó que los medios nativos recurrieron significativamente más al encuadre de candidata y experiencia política que los tradicionales, por eso se rechazó la $H 2$, que esperaba lo opuesto. Sin embargo, no hubo evidencia de que los encuadres de género, raza y novedad hayan sido destacados más por un tipo de medio que por otro.

\section{Discusión y conclusiones}

Respecto a los actores que aparecieron en la información noticiosa, es posible indicar que los medios le retiraron a Harris el protagonismo de la noticia para presentarla junto con otros actores, como Flores Márquez (2020) advierte sobre la cobertura de mujeres políticas. Esto podría dar la idea de que Harris logró la vicepresidencia gracias al nombramiento de Biden o por la animadversión de los ciudadanos ante Trump, en detrimento de su trayectoria o logros políticos (García Beaudoux et al., 2018; Power, 2017; Rista, 2020). Este hallazgo coincide con la perpetuación de la corresponsabilidad planteada por Ríos Sierra (2017), donde los logros de las candidatas se representan de manera compartida con otros hombres.

Al contrario de lo destacado en investigaciones que han encontrado una mediatización de la esfera íntima de mujeres candidatas (Flores Márquez, 2020; Foster Shoaf y Parsons, 2016; García Beaudoux et al., 2018; Rista, 2020; Van der Pas y Aaldering, 2020), el presente estudio detectó una baja presencia de los familiares de Harris, ya que apenas se mencionó al esposo Douglas Emhoff, a su hermana Maya Harris y a su madre Shyamala Gopalan. En ese sentido, los medios mexicanos prefirieron hacer la cobertura sobre Kamala Harris en relación con otros políticos, que con su círculo familiar cercano. 
En cuanto a la segunda pregunta de investigación acerca de la agenda temática, se concluye que los temas asociados con lo masculino, preferentemente política internacional, migración y economía, fueron más frecuentes que los temas asociados con lo femenino, como ciencia y cambio climático. Esto contradice lo que se ha encontrado en algunas investigaciones respecto a que hay una preponderancia de tópicos femeninos en las candidaturas de mujeres (Humprecht y Esser, 2017; Van der Pas y Aaldering, 2020; Waters et al., 2018). Lo anterior pudo deberse a que se trataba de una cobertura transnacional en un país que tiene frontera con Estados Unidos; por eso, los medios, más que ubicarla en temas femeninos o masculinos, hicieron la cobertura a partir de la manera en que podría impactar en México la elección de Kamala Harris como vicepresidenta.

No obstante, en lo que sí se coincide con los estudios anteriores, es que la cobertura de Harris - como mujer de ascendencia asiática y jamaiquina- se realizó con base en temas de racismo y migración. De acuerdo con la literatura consultada, esta actitud de los medios limitó el campo de acción de Harris, ya que por sus orígenes parecía obligada a opinar sobre temas raciales y de apoyo a su comunidad, fuera o no parte de su agenda política (Gershon y Lavariega Monforti, 2019; Maiolino, 2018; Wineinger, 2019).

En cuanto a la tercera pregunta de investigación acerca de cuáles fueron las maneras para referirse al origen racial de Harris, se hallaron al menos ocho formas. En este caso, se concluye que los medios la describieron como una candidata con multiplicidad de identidades. Esta complejidad relatada en las noticias es interesante sobre todo por los estudios que demuestran que las comunidades étnicas suelen votar por representantes políticos de su etnia, lo cual volvió a la candidata como parte de más de una comunidad étnica/racial y por ende con mayor potencial para atraer el voto de dichas comunidades (Block Jr., 2019; Johnson Carew, 2016; Mogahed y Mahmood, 2019). Por ello, se sugiere que los estudios futuros sobre identidades interseccionales hagan hincapié en si la información periodística reitera una sola o la multiplicidad de identidades.

A partir de la última pregunta de investigación, se obtuvieron dos conclusiones. Primero, que el tratamiento periodístico de las medios mexicanos estuvo centrado en mayor medida en el estatus de candidata y la experiencia política de Harris. Si bien la raza, el género y la novedad estuvieron presentes durante toda la campaña, éstos no fueron más frecuentes que los encuadres políticos. 
Dicho hallazgo va en contra de lo detectado por estudios anteriores que demostraban un ocultamiento de las credenciales políticas, para mostrarla preferentemente como un sujeto político de otredad en la arena política (Galy-Badenas y Gray, 2020; Lucas, 2017; Power, 2017; Rista, 2020; Towner y Clawson, 2016; Ward, 2016a y 2016b). En ese sentido, se coincide más con los trabajos que han encontrado una politización de la información noticiosa cuando abordan noticias internacionales (García Galindo y De Vicente Domínguez, 2014; Quevedo Redondo y Suárez Romero, 2017; RodríguezWangüemert et al., 2019).

No obstante, se recomienda que los encuadres de raza, género y novedad continúen siendo objeto de investigación académica, ya que si bien resultaron menos presentes en este trabajo, no fueron omitidos, porque aparecieron hasta en $50 \%$ de la información periodística y podrían ser utilizados por el electorado como atajos cognitivos para emitir su voto (García Beaudoux et al., 2020).

El segundo hallazgo respecto a esta última pregunta de investigación fue que los medios nativos digitales mostraron estadísticamente mayor cobertura de los encuadres políticos que los medios tradicionales, a pesar de que se esperaba lo contrario (Nielsen, 2020). En este tenor, se sugiere hacer comparaciones más extensas entre los medios nativos y los tradicionales para saber si la nueva forma de hacer periodismo en Internet ofrece una mirada más politizada de las candidatas interseccionales.

En términos generales, el trabajo creó un andamiaje teórico emergente sobre la forma en que pueden ser estudiadas las mujeres políticas con identidad interseccional en campañas electorales. Se recomienda que los estudios futuros se aboquen a examinar las distintas maneras de mencionar el origen racial y el balance en la cobertura informativa entre encuadres políticos y encuadres personales. No obstante, se sugiere revisar la pertinencia de utilizar este modelo en diversos contextos, mediante pruebas de fiabilidad sobre el instrumento propuesto, lo cual representó una limitante metodológica para el presente estudio. 
Convergencia Revista de Ciencias Sociales, vol. 28, 2021, Universidad Autónoma del Estado de México

\section{Referencias}

24 Horas (2021), 24 Horas. El diario sin limites. Disponible en: https://www.24-horas.mx [26 de enero de 2021].

Aaldering, Loes y Vliegenthart, Rens (2016), "Political leaders and the media. Can we measure political leadership images in newspapers using computer-assisted content analysis?”, en Quality \& Quantity, vol. 50, núm. 5, Países Bajos: Springer.

AFP y Reuters (2020), "Joe Biden gana la presidencia de Estados Unidos, en medio del coraje de Donald Trump", en El Economista. Disponible en: https://www.eleconomista.com. $\mathrm{mx} /$ internacionales/Joe-Biden-gana-la-presidencia-de-Estados-Unidos-en-medio-delcoraje-de-Donald-Trump-20201107-0008.html [8 de noviembre de 2020].

Alexander-Floyd, Nikol (2014), "Why political scientists don't study black women, but historians and sociologists do: on intersectionality and the remapping of the study of black political women", en Mitchell, Michael y Covin, David [eds.], Black Women in Politics. Identity, Power, and Justice in the New Millennium, Canadá: Transaction Publishers.

Álvarez-Monsiváis, Edrei (2019), “Tratamiento informativo de candidatas presidenciales: una propuesta desde el framing”, en Correspondencias \& Análisis, vol. 10, Perú: Universidad de San Martín de Porres.

Álvarez-Monsiváis, Edrei (2020), “De primera dama a candidata presidencial: masculinización en la cobertura periodística de mujeres políticas”, en Cuadernos.info, núm. 47, Chile: Pontifica Universidad Católica de Chile.

Animal Politico (2021), Periodismo libre para el ciudadano. Disponible en: https://www. animalpolitico.com [26 de enero de 2021].

Arbeláez Gómez, Martha Cecilia y Onrubia Goñi, Javier (2014), “Análisis bibliométrico y de contenido. Dos metodologías complementarias para el análisis de la revista colombiana Educación y Cultura”, en Revista de Investigaciones, vol. 14, núm. 23, Colombia: Universidad Católica de Manizales.

Ardèvol-Abreu, Alberto (2015), "Framing o teoría del encuadre en comunicación. Orígenes, desarrollo y panorama actual en España”, en Revista Latina de Comunicación Social, núm. 7, España: Sociedad Latina de Comunicación.

Aristegui Noticias (2021), Desde cualquier medio, periodismo en libertad. Disponible en: https://aristeguinoticias.com [26 de enero de 2021].

Baitinger, Gail (2015), "Meet the press or meet the men? Examining women's presence in American news media”, en Political Research Quarterly, vol. 68, núm. 3, Estados Unidos: Sage.

Barry, Ellen (2020), "La historia de amor de dos inmigrantes: cómo se conocieron los padres de Kamala Harris", en The New York Times. Disponible en: https://nyti.ms/35QUk4X [30 de noviembre de 2020].

Bashri, Maha (2019), "Elections, representations, and journalistic schemas: local news coverage of Ilhan Omar and Rashida Tlaib in the US mid-term elections", en ESSACHESS Journal for Communication Studies, vol. 12, núm. 24, Francia: University Paul Valery of Montpellier 3.

Bernete, Francisco (2013), "Análisis de contenido (cuantitativo y cualitativo", en Lucas Marín, Antonio y Noboa, Alejandro [eds.], Conocer lo social: Estrategias y técnicas de construcción y análisis de datos, España: Fragua. 
Biden, Joe (2020), “I have the great honor to announce that I've picked @KamalaHarris - a fearless fighter for the little guy, and one" [tweet], https://twitter.com/JoeBiden/ status/1293280411150217219.

Bleich, Erik et al. (2015), "Media portrayals of minorities. Muslims in British newspaper headlines, 2001-2012", en Journal of Ethnic and Migration Studies, vol. 41, núm. 6, Reino Unido: Routledge.

Block Jr., Ray (2017), "Race, gender, and media coverage of Michelle Obama", en Politics, Groups and Identities, vol. 5, núm. 1, Reino Unido: Routledge.

Block Jr., Ray (2019), “Racial stereotyping in political decision making”, en Oxford Research Encyclopedia of Politics, Reino Unido: Oxford University Press.

Boumans, Jelle y Trilling, Damian (2016), "Taking stock of the toolkit. An overview of relevant automated content analysis approaches and techniques for digital journalism scholars”, en Digital Journalism, vol. 4, núm. 1, Reino Unido: Routledge.

Brown, Nadia y Gershon, Sarah Allen (2016), "Intersectional presentations: an exploratory study of minority congresswomen's websites' biographies", en Du Bois Review: Social Science Research on Race, vol. 13, núm. 1, Reino Unido: Cambridge University Press.

Brown, Nadia y Gershon, Sarah Allen (2020), "Glass half full: cautious optimism and the future of black women political elites in America", en Journal of Race, Ethnicity and Politics, Reino Unido: Cambridge University Press.

Carrasco-Campos, Ángel y Saperas, Enric (2015), “The operationalization of the concept of framing in the 'Journal of Communication' (2009-2013): objects of study, research techniques and theoretical construction", en Communication \& Society, vol. 28, núm. 4, España: Universidad de Navarra.

Catalán-Matamoros, Daniel y Peñafiel-Saiz, Carmen (2019), "Medios y desconfianza en vacunas: un análisis de contenido en titulares de prensa”, en Revista Latina de Comunicación Social, núm. 74, España: Sociedad Latina de Comunicación.

Collins, Patricia (1990), Black Feminist Thought: Knowledge, Consciousness and the Politics of Empowerment, Reino Unido: Routledge.

Cordero, Álvaro (2020), "El Colegio Electoral confirmó la victoria de Joe Biden en las elecciones presidenciales de EE. UU., en France 24. Disponible en: https://www. france24.com/es/ee-uu-y-canadá/20201214-elecciones-eeuu-biden-presidentetrump-colegio-electoral [30 de noviembre de 2020].

Crenshaw, Kimberlé (1991), "Mapping the margins: intersectionality, identity politics, and violence against women of color", en Stanford Law Review, vol. 43, núm 6, Estados Unidos: Stanford University.

Cultura Colectiva (2021), Medio de difusión cultural y generación de contenido. Disponible en: https://culturacolectiva.com [26 de enero de 2021].

D’Angelo, Paul y Shaw, Donna (2018), "Journalism as framing", en Vos, Tim [ed.], Journalism, Alemania: Gruyter Mouton.

Díaz Herrera, Claudio (2018), "Investigación cualitativa y análisis de contenido temático. Orientación intelectual de revista Universum", en Revista General de Información y Documentación, vol. 28, núm. 1, España: Universidad Complutense de Madrid.

El Financiero (2021), Las noticias de finanzas, economía y politica más importantes del país. Disponible en: https://www.elfinanciero.com.mx [26 de enero de 2021].

El Heraldo (2021), El diario que piensa joven. Disponible en: https://heraldodemexico.com. $\mathrm{mx}$ [26 de enero de 2021]. 
Convergencia Revista de Ciencias Sociales, vol. 28, 2021, Universidad Autónoma del Estado de México

El Sol de México (2021), Noticias, deportes, gossip, columnas. El Sol de México. Disponible en: https://www.elsoldemexico.com.mx [26 de enero de 2021].

El Universal (2021), El periódico de México lider en noticias y clasificados. Disponible en: https://www.eluniversal.com.mx [26 de enero de 2021].

Evans, Elizabeth (2015), “Diversity matters: intersectionality and women's representation in the USA and UK”, en Parliamentary Affairs, vol. 69, núm. 3, Reino Unido: Oxford University Press.

Excélsior (2021), El periódico de la vida nacional. Disponible en: https://www.excelsior. com.mx [26 de enero de 2021].

Flores Márquez, Dorismilda (2020), "La dimension comunicacional: Desigualdad de género en la presencia mediática de las candidaturas”, en Góngora Cervantes, Verónica et al. [eds.], Violencia politica electoral contra las mujeres en Guanajuato. Análisis del proceso 2017-2018, México: Instituto Electoral del Estado de Guanajuato.

Foster Shoaf, Nicole y Parsons, Tara (2016), “18 million cracks, but no cigar: news media and the campaigns of Clinton, Palin, and Bachmann”, en Social Sciences, vol. 5, núm. 3, Estados Unidos: Multidisciplinary Digital Publishing Institute.

Galy-Badenas, Flora y Gray, Elizabeth (2020), "Media coverage of Rachida Dati and Najat Vallaud-Belkacem: an intersectional analysis of representations of minority women in the French political context", en Women's Studies in Communication, vol. 43, núm. 2, Reino Unido: Taylor and Francis.

García Beaudoux, Virgina et al. (2020), "Estereotipos y sesgos en el tratamiento de candidatas y candidatos en programas televisivos en las elecciones legislativas de Argentina en 2017”, en Revista Latina de Comunicación Social, núm. 77, España: Sociedad Latina de Comunicación.

García Beaudoux, Virginia et al. (2018), "Una tipología de los sesgos y estereotipos de género en la cobertura periodística de las mujeres candidatas", en Revista Mexicana de Opinión Pública, vol. 13, núm. 24, México: Universidad Nacional Autónoma de México.

García Galera, María del Carmen y Berganza Conde, María Rosa (2005), "El método científico aplicado a la investigación en comunicación mediática”, en Berganza Conde, María Rosa y Ruiz San Román, José [eds.], Investigar en Comunicación: Guía Práctica de Métodos y Técnicas de Investigación Social en Comunicación, España: McGraw-Hill.

García Galindo, Juan Antonio y De Vicente Domínguez, Aída María (2014), "Análisis de la información internacional en la prensa digital española”, en Estudios sobre el Mensaje Periodístico, vol. 20, núm. 1, España: Universidad Complutense de Madrid.

García-Peter, Sabina y Villavicencio-Miranda, Luis (2016), “Alcances y límites del multiculturalismo liberal desde un enfoque de género interseccional”, en Convergencia. Revista de Ciencia Sociales, núm. 72, México: Universidad Autónoma del Estado de México.

Gerrits, Bailey et al. (2017), "Political battlefield: aggressive metaphors, gender, and power in news coverage of Canadian party leadership contests", en Feminist Media Studies, vol. 17, núm. 6, Reino Unido: Routledge.

Gershon, Sarah Allen (2012), "When race, gender, and the media intersect: campaign news coverage of minority congresswomen”, en Journal of Women, Politics \& Policy, vol. 33, núm. 2, Estados Unidos: Routledge.

Gershon, Sarah Allen y Lavariega Monforti, Jessica (2019), "Intersecting campaigns: candidate race, ethnicity, gender and voter evaluations", en Politics, Groups, and Identities, Estados Unidos: Routledge. 
González-Teruel, Aurora (2015), “Estrategias metodológicas para la investigación del usuario en los medios sociales: análisis de contenido, teoría fundamentada y análisis del discurso", en Profesional de la Información, vol. 24, núm. 3, España: Profesional de la Información.

Guzmán Ordaz, Raquel (2015), “El paradigma interseccional: rutas teórico-metodológicas para el análisis de las desigualdades sociales”, en Saletti Cuesta, Lorena [ed.], Traslaciones en los Estudios Feministas, España: Perséfone.

Haraldsson, Amanda y Wängnerud, Lena (2019), “The effect of media sexism on women's political ambition: evidence from a worldwide study", en Feminist Media Studies, vol. 19, núm. 4, Reino Unido: Routledge.

Haynes, Christina y Block Jr., Ray (2019). "Role-model-in-chief: understanding a Michelle Obama effect”, en Politics \& Gender, vol. 15, núm. 3, Reino Unido: Cambridge University Press.

Humprecht, Edda y Esser, Frank (2017), "A glass ceiling in the online age? Explaining the underrepresentation of women in online political news", en European Journal of Communication, vol. 32, núm. 5, Reino Unido: Sage.

INE (2020), Catálogo nacional de medios impresos e internet 2020. Disponible en: https:// repositoriodocumental.ine.mx/xmlui/bitstream/handle/123456789/113534/ JGEor202002-17-ap-3-1-a.pdf [8 de noviembre de 2020].

Johnson Carew, Jessica (2016), "How do you see me?: stereotyping of black women and how it affects them in an electoral context", en Brown, Nadia y Gershon, Sarah Allen [eds.], Distinct Identities. Minority Women in U.S. Politics, Estados Unidos: Routledge.

Kwon, Eunseon y Frisby, Cynthia (2015), "Readings and research about diversity across audiences and media content", en Frisby, Cynthia [ed.], How You See Me, How You Don't: Essays on Stereotypes and Representations in Media of Minorities, Adolescents, and Women, Estados Unidos: Tate Publishing.

La Jornada (2021), La Jornada. Disponible en: https://www.jornada.com.mx [26 de enero de 2021].

La Silla Rota (2021), Noticias esenciales de México. Disponible en: https://lasillarota.com [26 de enero de 2021].

Lachover, Einat (2017), "Signs of change in media representation of women in Israeli politics: leading and peripheral women contenders", en Journalism, vol. 18, núm. 4, Estados Unidos: Sage.

Lima, Alice y Panke, Luciana (2016), “Tipologias de gênero feminino no discurso eleitoral. O caso da esquerda brasileira em 2014”, en Cuestiones de Género: de la Igualdad y la Diferencia, núm. 11, España: Universidad de León.

López-Dóriga (2021), Las últimas noticias de México y el mundo. Disponible en: https:// lopezdoriga.com [26 de enero de 2021].

Lucas, Jennifer (2017), "Gender and race in congressional national news media appearances in 2008”, en Politics \& Gender, vol. 13, núm. 4, Reino Unido: Cambridge University Press.

Lyall, Sarah (2020), “What kind of Second Gentleman would Doug Emhoff be?”, en The New York Times. Disponible en: https://nyti.ms/2E6GzUk [30 de noviembre de 2020].

Maiolino, Elise (2018), “'I'm not a male, not white, want to start there?': Olivia Chow and identity work in Toronto's 2014 mayoral election”, en Journal of Women, Politics \& Policy, vol. 39, núm. 2, Estados Unidos: Routledge. 
Convergencia Revista de Ciencias Sociales, vol. 28, 2021, Universidad Autónoma del Estado de México

Marañón Lazcano, Felipe et al. (2018), "La mujer política en Twitter: análisis de los mensajes emitidos por las candidatas a gubernaturas en México”, en adComunica, núm. 16, España: Universitat Jaume I.

Mars, Amanda (2019), "La demócrata Kamala Harris se retira de la carrera presidencial para 2020”, El Pais. Disponible en: https://elpais.com/internacional/2019/12/03/ estados_unidos/1575397223_571175.html [30 de noviembre de 2020].

Matthes, Jörg (2009), "What's in a frame? A content analysis of media framing studies in the world's leading communication journals, 1990-2005", en Journalism \& Mass Communication Quarterly, vol. 86, núm. 2, Estados Unidos: Sage.

Merchant, Iván (2020), "6 medios de noticias consiguen un sitio en el top 10 de octubre", en El Economista. Disponible en: https://www.eleconomista.com.mx/tecnologia/6medios-de-noticias-consiguen-un-sitio-en-el-top-10-de-octubre-20201127-0051. html [ 8 de noviembre de 2020].

Meyers, Marian y Goman, Carmen (2017), “Michelle Obama: exploring the narrative”, en Howard Journal of Communications, vol. 28, núm. 1, Reino Unido: Taylor and Francis.

Milenio (2021), Noticias de hoy en México y el mundo. Disponible en: https://www.milenio. com [26 de enero de 2021].

Mitchelstein, Eugenia et al. (2019), "Joanne Public vs. Joe Public: news sourcing and gender imbalance on Argentine digital media”, en Digital Journalism, vol. 7, núm. 10, Reino Unido: Taylor and Francis.

Mogahed, Dalia y Mahmood, Azka (2019), “American Muslim poll 2019. Predicting and preventing islamophobia”, en Institute for Social Policy and Understanding. Disponible en: https://www.ispu.org/american-muslim-poll-2019-predicting-and-preventingislamophobia/ [30 de noviembre de 2020].

Muñiz, Carlos (2020), "El framing como proyecto de investigación: una revisión de conceptos, ámbitos y métodos de estudio", en Profesional de la Información, vol. 29, núm. 6, España: Profesional de la Información.

Nielsen, Carolyn (2020), "Send her back: news narratives, intersectionality, and the rise of politically powerful women of color", en International Symposium on Online Journalism, vol. 10, núm. 1, Estados Unidos: Knight Center.

Owens, Donna (2016), "Meet Kamala Harris, the second Black women elected to the U.S. Senate”, en NBC News. Disponible en: https://www.nbcnews.com/storyline/2016election-day/meet-kamala-harris-second-black-woman-elected-u-s-senate-n680726 [30 de noviembre de 2020].

Panke, Luciana (2015), Campañas electorales para mujeres: retos y tendencias, México: Piso 15.

Panke, Luciana et al. (2015), "Género y campañas electorales en América Latina. Un análisis del discurso femenino en la propaganda televisiva”, en Razón y Palabra, núm. 91, México, Ecuador y Colombia: Tecnológico de Monterrey, Pontificia Universidad Católica del Ecuador y Universidad de Bogotá Jorge Tadeo Lozano.

Piñeiro-Naval, Valeriano y Mangana, Rafael (2018), “Teoría del encuadre: panorámica conceptual y estado del arte en el contexto hispano", en Estudios sobre el Mensaje Periodístico, vol. 24, núm. 1, España: Universidad Complutense de Madrid.

Piñeiro-Naval, Valeriano y Morais, Ricardo (2020), "La política como temática de estudio en las revistas hispánicas de comunicación”, en Convergencia Revista de Ciencias Sociales, vol. 27, México: Universidad Autónoma del Estado de México. 
Power, Kate (2017), "Church trailblazer Rev Pat Storey on weight watchers, caffeine and how she named her dog after former New York Mayor: news representations of the first female Anglican Bishop in the UK and Ireland", en Irish Journal of Applied Social Studies, vol. 16, núm. 1, Irlanda: Social Care Ireland.

Publimetro (2021), Noticias, deportes y entretención de México y el mundo. Disponible en: https://www.publimetro.com.mx/mx/ [26 de enero de 2021].

Quevedo Redondo, Raquel y Suárez-Romero, Miriam (2017), "Del retrato de la Dama de Hierro a la fotografía de Mutti Angela: la representación icónica de la canciller alemana Angela Merkel”, en Estudios sobre el Mensaje Periodístico, vol. 23, núm. 1, España: Universidad Complutense de Madrid.

Reforma (2021), Periodismo independiente. Disponible en: https://www.reforma.com [26 de enero de 2021].

Reporte Índigo (2021), Reporte Índigo. Disponible en: https://www.reporteindigo.com [26 de enero de 2021].

Rincón Soto, Lucía (2015), "Mujer y poder político en Latinoamérica: Una reflexión desde el siglo XXI", en Utopia y Praxis Latinoamericana, vol. 20, núm. 68, Venezuela: Universidad del Zulia.

Ríos Sierra, Jerónimo (2017), “Liderazgo político y patriarcado mediático: las imágenes políticas de Cristina Fernández y Dilma Rousseff”, en Revista de Investigaciones Politicas y Sociológicas, vol. 16, núm. 1, España: Universidad de Santiago de Compostela.

Rista, Micaela (2020), "Framing y estereotipos de género en medios de comunicación: los casos de Cristina Fernández de Kirchner y María Eugenia Vidal en la campaña electoral 2019”, en Sociales Investiga, vol. 3, núm. 6, Argentina: Universidad Nacional Villa María.

Rodelo, Frida (2016), “Disparidades de género en la cobertura mediática de campañas electorales locales en México", en Cuadernos.info, núm. 39, Chile: Pontificia Universidad Católica de Chile.

Rodelo, Frida (2020), “Las candidatas en los medios de comunicación”, en Rangel Juárez, Griselda Beatriz et al. [eds.], Más Allá de la Paridad. Elecciones 2018 en Jalisco, México: Universidad de Guadalajara.

Rodríguez-Wangüemert, Carmen et al. (2019), "Encuadres de China en la televisión española”, en Communication \& Society, vol. 32, núm. 3, España: Universidad de Navarra.

SDP Noticias (2021), Últimas noticias de México y el mundo en SDPnoticias.com. Disponible en: https://www.sdpnoticias.com [26 de enero de 2021].

Sensales, Gilda et al. (2016), "Linguistic sexism in the news coverage of women ministers from four Italian governments: an analysis from a social-psychological perspective”, en Journal of Language and Social Psychology, vol. 35, núm. 4, Estados Unidos: Sage.

Showunmi, Victoria et al. (2016), "Ethnic, gender and class intersections in British women's leadership experiences", en Educational Management Administration \& Leadership, vol. 44, núm. 6, Reino Unido: Sage.

Sin Embargo (2021), Periodismo digital con rigor. Disponible en: https://www.sinembargo. $\mathrm{mx}$ [26 de enero de 2021].

Snipes, Alexandra y Mudde, Cas (2019), “'France’s (kinder, gentler) extremist': Marine Le Pen, intersectionality, and media framing of female populist radical right leaders", en Politics \& Gender, vol. 16, núm. 2, Reino Unido: Cambridge University Press. 
Sopitas (2021), Sopitas.com. Disponible en: https://www.sopitas.com [26 de enero de 2021]. Tolley, Erin (2015), Framed: Media and the Coverage of Race in Canadian Politics, Canadá: University of British Columbia Press.

Towner, Terri y Clawson, Rosalee (2016), "A wise Latina or a baffled rookie? Media coverage of justice Sonia Sotomayor's ascent to the bench", en Journal of Women, Politics \& Policy, vol. 37, núm. 3, Estados Unidos: Routledge.

UnoTV (2021), Últimas noticias de hoy en México y el mundo en vivo. Disponible en: https:// www.unotv.com [26 de enero de 2021].

Van der Pas, Daphne y Aaldering, Loes (2020), "Gender differences in political media coverage: a meta-analysis", en Journal of Communication, vol. 70, núm. 1, Estados Unidos: Wiley-Blackwell.

Vidal Correa, Fernanda (2020a), "Presidential elections in Mexico: media coverage of the Josefina Vazquez Mota campaign", en Women's Studies, vol. 49, núm. 5, Estados Unidos: Routledge.

Vidal Correa, Fernanda (2020b), "Media coverage of campaigns: a multilevel study of Mexican women running for office", en Communication \& Society, vol. 33, núm. 3, España: Universidad de Navarra.

Vidal Correa, Fernanda (2020c), "Media coverage of women in politics: Mexican local politicians on campaign", en The Journal of International Communication, vol. 26, núm. 1, Estados Unidos y Reino Unido: Routledge.

Wagner, Angelia et al. (2019), "One smart politician: gendered media discourses of political leadership in Canada”, en Canadian Journal of Political Science, vol. 52, núm. 1, Reino Unido: Cambridge University Press.

Ward, Orlanda (2016a), "Intersectionality and press coverage of political campaigns: representations of black, Asian, and minority ethnic female candidates at the U.K. 2010 General Election", en The International Journal of Press/Politics, vol. 22, núm. 1, Estados Unidos: Sage.

Ward, Orlanda (2016b), "Seeing double: race, gender, and coverage of minority women's campaigns for the U.S. House of Representatives", en Politics \& Gender, vol. 12, núm. 2, Reino Unido: Cambridge University Press.

Waters, Susan et al. (2018), "Battleground Texas: gendered media framing of the 2014 Texas gubernatorial race”, en Journal of Feminist Scholarship, núm. 14, Estados Unidos: University of Massachusetts Dartmouth.

Wineinger, Catherine (2019), "How can a black woman be a Republican? An intersectional analysis of identity claims in the 2014 Mia Love campaign", en Politics, Groups, and Identities, Estados Unidos: Routledge. 


\section{Anexos}

\section{Tabla 1}

Noticias recolectadas de acuerdo con los medios analizados

Sitios web de medios impresos

\section{Visitantes}

mensuales Noticias

Sitio (millones)

El Universal

39

El Heraldo

Milenio

La Jornada

Excélsior

Reforma

24 Horas

El Sol de

México

El Financiero

Publimetro

Totales

32

25

20

16

11

4,8

4,7

4,5

49 analizadas Sitio

UnoTV

32 SDP Noticias

27 Animal Politico

7 Cultura Colectiva

23 La Silla Rota

18 Reporte Índigo

23 Aristegui Noticias

15 Sin Embargo

3,2

1,4

1,4

48,5 $\quad(\mathrm{n}=107)$ (millones) 17 8,2 3,7 3,6 3,4 3,3

3,3 analizadas

17 5 4 5 19 4 14
Visitantes mensuales

totales 209,7 millones

Noticias recolectadas totales $\mathrm{N}=329$
4,2

13 López-Dóriga $(\mathrm{n}=$

161,2 222) Totales mensuales Noticias

totales

Fuente: Elaboración propia con datos del INE (2020) y Merchant (2020). 


\section{Tabla 2}

Actores que aparecen en la noticia junto con Kamala Harris

\begin{tabular}{|c|c|c|c|c|c|c|}
\hline \multirow[b]{2}{*}{ Actores } & \multicolumn{2}{|c|}{ Nativos } & \multicolumn{2}{|c|}{ Tradicionales } & \multicolumn{2}{|c|}{ Totales } \\
\hline & $\mathrm{f}$ & $\mathrm{M}$ & $\mathrm{f}$ & $\mathrm{M}$ & $\mathrm{f}$ & $\mathrm{M}$ \\
\hline Joe Biden & 103 & 0,96 & 208 & 0,94 & 311 & 0,95 \\
\hline Donald Trump & 86 & 0,80 & 178 & 0,80 & 264 & 0,80 \\
\hline Mike Pence & 35 & 0,33 & 75 & 0,34 & 110 & 0,33 \\
\hline Barack Obama & 41 & 0,38 & 56 & 0,25 & 97 & 0,29 \\
\hline Hillary Clinton & 13 & 0,12 & 32 & 0,14 & 45 & 0,14 \\
\hline George Floyd & 9 & 0,08 & 24 & 0,11 & 33 & 0,10 \\
\hline Douglas Emhoff & 9 & 0,08 & 16 & 0,07 & 25 & 0,08 \\
\hline Sarah Palin & 4 & 0,04 & 20 & 0,09 & 24 & 0,07 \\
\hline Susan Page & 6 & 0,06 & 16 & 0,07 & 22 & 0,07 \\
\hline Brett Kavanaugh & 4 & 0,04 & 17 & 0,08 & 21 & 0,06 \\
\hline Maya Harris & 10 & 0,09 & 11 & 0,05 & 21 & 0,06 \\
\hline Nancy Pelosi & 3 & 0,03 & 16 & 0,07 & 19 & 0,06 \\
\hline $\begin{array}{l}\text { Shyamala } \\
\text { Gopalan }\end{array}$ & 9 & 0,08 & 10 & 0,05 & 19 & 0,06 \\
\hline Otros / Ninguno & 1 & 0,01 & 2 & 0,01 & 3 & 0,01 \\
\hline Totales & \multicolumn{2}{|c|}{$(\mathrm{n}=107)$} & \multicolumn{2}{|c|}{$(n=222)$} & \multicolumn{2}{|c|}{$(\mathrm{N}=329)$} \\
\hline
\end{tabular}

Nota: Cada noticia pudo haber presentado más de un actor.

Fuente: Elaboración propia con base en las noticias analizadas. 


\section{Tabla 3}

\section{Temáticas de las noticias}

\begin{tabular}{|c|c|c|c|c|c|c|}
\hline \multirow[b]{2}{*}{ Tópicos } & \multicolumn{2}{|c|}{ Nativos } & \multicolumn{2}{|c|}{ Tradicionales } & \multicolumn{2}{|c|}{ Totales } \\
\hline & $\mathrm{f}$ & $\mathrm{M}$ & $\mathrm{f}$ & M & $\mathrm{f}$ & $\mathrm{M}$ \\
\hline Pandemia Covid-19 & 58 & 0,54 & 114 & 0,51 & 172 & 0,52 \\
\hline Política internacional y migración & 41 & 0,38 & 104 & 0,47 & 145 & 0,44 \\
\hline Racismo & 47 & 0,44 & 92 & 0,41 & 139 & 0,42 \\
\hline Debates electorales & 41 & 0,38 & 91 & 0,41 & 132 & 0,40 \\
\hline Resultados electorales & 44 & 0,41 & 66 & 0,30 & 110 & 0,33 \\
\hline Economía & 44 & 0,41 & 65 & 0,29 & 109 & 0,33 \\
\hline Derechos civiles y minorías & 27 & 0,25 & 72 & 0,32 & 99 & 0,30 \\
\hline Convención demócrata & 16 & 0,15 & 27 & 0,12 & 43 & 0,13 \\
\hline Ciencia & 12 & 0,11 & 16 & 0,07 & 27 & 0,08 \\
\hline Cambio climático & 9 & 0,08 & 17 & 0,08 & 25 & 0,08 \\
\hline Otros & 2 & 0,02 & 16 & 0,07 & 18 & 0,05 \\
\hline Totales & \multicolumn{2}{|c|}{$(\mathrm{n}=107)$} & \multicolumn{2}{|c|}{$(\mathrm{n}=222)$} & \multicolumn{2}{|c|}{$(\mathrm{N}=329)$} \\
\hline
\end{tabular}

Nota: Cada noticia pudo haber sido clasificada en más de un tópico.

Fuente: Elaboración propia con base en las noticias analizadas. 


\section{Tabla 4}

Formas de referirse al origen racial de Kamala Harris

\begin{tabular}{|c|c|c|c|c|c|c|}
\hline \multirow[b]{2}{*}{ Encuadre de raza } & \multicolumn{2}{|c|}{ Nativos } & \multicolumn{2}{|c|}{ Tradicionales } & \multicolumn{2}{|c|}{ Totales } \\
\hline & $\mathrm{f}$ & $\mathrm{M}$ & $\mathrm{f}$ & $\mathrm{M}$ & $\mathrm{f}$ & $\mathrm{M}$ \\
\hline Origen de los padres & 53 & 0,82 & 106 & 0,85 & 159 & 0,84 \\
\hline Negra & 32 & 0,49 & 62 & 0,50 & 94 & 0,50 \\
\hline Afroestadounidense & 32 & 0,49 & 52 & 0,42 & 84 & 0,44 \\
\hline Asiática & 20 & 0,31 & 39 & 0,31 & 59 & 0,31 \\
\hline Origen indio & 17 & 0,26 & 15 & 0,12 & 32 & 0,17 \\
\hline De color & 10 & 0,15 & 17 & 0,14 & 27 & 0,14 \\
\hline Origen jamaiquino & 4 & 0,06 & 4 & 0,03 & 8 & 0,04 \\
\hline Raza mixta o mestiza & 3 & 0,05 & 3 & 0,02 & 6 & 0,03 \\
\hline Totales & \multicolumn{2}{|c|}{$(\mathrm{n}=65)$} & \multicolumn{2}{|c|}{$(\mathrm{n}=124)$} & \multicolumn{2}{|c|}{$(\mathrm{n}=189)$} \\
\hline
\end{tabular}

Nota: Cada noticia pudo haber presentado más de una referencia a su origen. Fuente: Elaboración propia con base en las noticias analizadas.

\section{Tabla 5}

Presencia de los encuadres en las noticias en función del tipo de medio digital

\begin{tabular}{|c|c|c|c|c|c|c|c|}
\hline \multirow[b]{2}{*}{ Encuadre } & \multicolumn{2}{|c|}{ Nativos } & \multicolumn{2}{|c|}{ Tradicionales } & \multicolumn{2}{|c|}{ Totales } & \multirow[b]{2}{*}{$\mathrm{d}$ de Cohen } \\
\hline & M & $\mathrm{DE}$ & M & $\mathrm{DE}$ & M & $\mathrm{DE}$ & \\
\hline Candidata & 0,92 & 0,28 & 0,85 & 0,36 & 0,87 & 0,33 & 0,21 \\
\hline Experiencia política & 0,75 & 0,44 & 0,65 & 0,48 & 0,68 & 0,47 & 0,22 \\
\hline Género & 0,57 & 0,50 & 0,61 & 0,49 & 0,60 & 0,49 & 0,08 \\
\hline Raza & 0,61 & 0,49 & 0,56 & 0,50 & 0,57 & 0,50 & 0,10 \\
\hline \multirow[t]{2}{*}{ Novedad } & 0,52 & 0,50 & 0,53 & 0,50 & 0,53 & 0,50 & 0,02 \\
\hline & \multicolumn{2}{|c|}{$(\mathrm{n}=107)$} & \multicolumn{2}{|c|}{$(\mathrm{n}=222)$} & \multicolumn{3}{|c|}{$(\mathrm{N}=329)$} \\
\hline
\end{tabular}

Nota: Los encuadres tenían un rango de variación de 0 (ausente) a 1 (presente) en cada noticia analizada.

Fuente: Elaboración propia con base en las noticias analizadas. 
Edrei Álvarez-Monsiváis. Doctor en Estudios Humanísticos, con énfasis en comunicación y medios, Universidad Autónoma de Nuevo León. Líneas de investigación: estudios de género, comunicación política y periodismo. Publicaciones recientes: Álvarez-Monsiváis, Edrei (2020), "Encuadres noticiosos sobre primeras damas: las expertas, las novatas y las actuales", en Profesional de la Información, vol. 29, núm. 6, España: Profesional de la Información; Álvarez Monsiváis, Edrei (2020), "De primera dama a candidata presidencial: masculinización en la cobertura periodística de mujeres políticas", en Cuadernos.info, núm. 47, Chile: Pontificia Universidad Católica de Chile; Álvarez-Monsiváis, Edrei (2020), "Narrativa web en las noticias sobre celebridades: sintaxis multimedia y redes sociales como fuente de información”, en Estudios sobre el Mensaje Periodístico, vol. 26, núm. 1, España: Universidad Complutense de Madrid. 